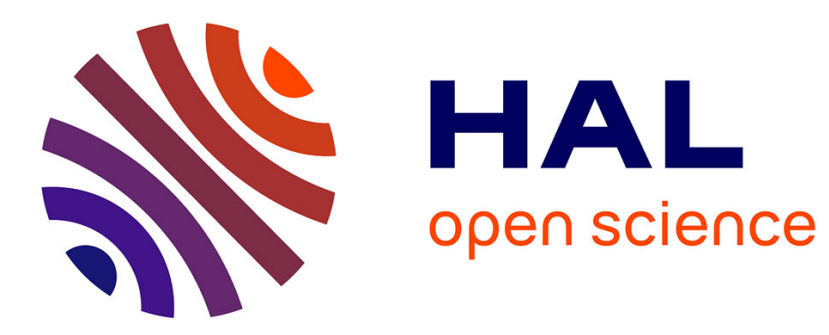

\title{
Securing Color Information of an Image by Concealing the Color Palette
}

Marc Chaumont, William Puech, Christian Lahanier

\section{To cite this version:}

Marc Chaumont, William Puech, Christian Lahanier. Securing Color Information of an Image by Concealing the Color Palette. Journal of Systems and Software, 2013, 86 (3), pp.809-825. 10.1016/j.jss.2012.11.042 . lirmm-00807065

\section{HAL Id: lirmm-00807065 https://hal-lirmm.ccsd.cnrs.fr/lirmm-00807065}

Submitted on 2 Apr 2013

HAL is a multi-disciplinary open access archive for the deposit and dissemination of scientific research documents, whether they are published or not. The documents may come from teaching and research institutions in France or abroad, or from public or private research centers.
L'archive ouverte pluridisciplinaire HAL, est destinée au dépôt et à la diffusion de documents scientifiques de niveau recherche, publiés ou non, émanant des établissements d'enseignement et de recherche français ou étrangers, des laboratoires publics ou privés. 


\title{
Securing Color Information of an Image by Concealing the Color Palette
}

\author{
M. Chaumont ${ }^{a, b, c}$, W. Puech ${ }^{b, c}$ and C. Lahanier ${ }^{d}$ \\ ${ }^{a}$ UNIVERSITE DE NIMES, F-30021 Nîmes, France \\ ${ }^{b}$ UNIVERSITE MONTPELLIER 2, UMR5506-LIRMM, F-34095 Montpellier, France \\ ${ }^{c}$ CNRS, UMR5506-LIRMM, F-34392 Montpellier, France \\ ${ }^{d} C 2 R M F$, UMR 171 CNRS Culture Ministry, Paris, France
}

\begin{abstract}
This paper deals with a method to protect the color information of images by providing free access to the corresponding gray level images. Only with a secret key and the gray level images, it is then possible to view the images in color. The approach is based on a color reordering algorithm after a quantization step. Based on a layer scanning algorithm, the color reordering generates gray level images and makes it possible to embed the color palette into the gray level images using a data hiding algorithm. This work was carried out in the framework of a project aimed at providing limited access to the private digital painting database of the Louvre Museum in Paris, France.
\end{abstract}

Keywords: Data hiding, Color protection, Color palette scanning, Palette reordering, Digital painting, Cultural heritage.

\section{Introduction}

Internet traffic is growing rapidly due to the massive use of multimedia data streams. The widespread transmission of images and videos often makes it necessary to find ways to secure them. As an example, applications like confidential transmission, video surveillance, cultural heritage, military and medical applications (Bernarding et al., 2001; Norcen et al., 2003) need security functionalities. Data security has thus become an important and integral component of modern day research on multimedia information.

Two main approaches have been developed for secure data transmission. The first one is based on content protection through encryption. There are several methods that encrypt binary or gray level images (Chung and Chang, 1998; Chang et al., 2001; Sinha and Singh, 2003; Uhl and Pommer, 2005). In this group, proper decryption of data requires a key. The second group bases the protection on digital watermarking or data hiding, with the aim of embedding a message into the data (Eskicioglu and Delp, 2001; Shih and Wu, 2003). These two technologies can be complementary (Xu et al., 2004; Lemma et al., 2006; Puech and Coatrieux, 2008) and mutually commutative (Lian et al., 2006). Sinha and Singh proposed an encryption technique that adds the digital signature of the original image to the encoded version of the original image (Sinha and Singh, 2003). The image encoding is carried out using an appropriate error control code. At the receiver end, after decryption of the image, the digital signature can be used to verify

Email address: marc.chaumont@lirmm.fr william.puech@lirmm.fr, christian.lahanier@free.fr(M. Chaumont $^{a, b, c}$, W. Puech ${ }^{b, c}$ and C. Lahanier ${ }^{d}$ )

URL: www. lirmm.fr $/^{\sim}$ chaumont (M. Chaumont ${ }^{a, b, c}$, W. Puech ${ }^{b, c}$ and C. Lahanier ${ }^{d}$ ) the authenticity of the image. An analysis of the local standard deviation of watermarked encrypted images has been proposed in (Puech et al., 2008) in order to remove embedded data during the decryption step. Irrespective of the approach chosen, data protection methods that depend on the algorithm secrecy are not considered to be true data protection methods (Schneier, 1995). In compliance with the Kerckhoffs principle (Kerckhoffs, 1883), existing encryption and data hiding methods are based on secret keys and not on the secrecy of the algorithms.

This paper presents a solution to provide free Internet access to low quality images to all users and restricted access to the same images of better quality with the purchase of a key. More precisely, the proposed solution tends to give free access to gray level images whereas the user needs a key to view the corresponding image in color. The main objective is thus to protect the color information of an image by embedding it in its corresponding gray level image. Users do not need to download the color image once the key is bought but users can only view the color image with the help of the gray level image and key. The proposed method consists of three major steps which involve quantization of the color image, color reordering by finding an index image and data-hiding in order to hide the color information in the index image. The first step consists of constructing a color palette of $K \in \mathbb{N}^{*}$ colors which well represents the color information of the 24-bit color image. The aim of the second step is to find an index image which is very close to the gray level image and the final step is to embed the color palette into the index image while ensuring the minimum possible distortion. This work was carried out in the framework of a project to give limited access to the private digital painting database of the Louvre Museum in Paris, France ${ }^{1}$. There has been greater interest in processing artwork images in the last five years, and

\footnotetext{
${ }^{1}$ The access to very high quality images is not allowed by the Louvre $\mathrm{Mu}-$
} 
a state of art is described in (Cappellini and Piva, 2006).

Previous work proposed to combine color image processing and data protection. In fact, these previous solutions involved hiding information by using the decomposition of a color image into an index image and a color palette. For example, datahiding may occur in the index image (Fridrich, 1999) or in the color palette (Wu et al., 2003; Tzeng et al., 2004). In (Wu et al., 2003) and (Tzeng et al., 2004) color palettes are used to secretly hide a message. In particular, Wu et al. proposed to build a new palette in order to embed one message bit into each color of the palette (Wu et al., 2003).

Other work, such as (Campisi et al., 2002; Zhao et al., 2004; R. De Queiroz and K. Braun, 2006; R. De Queiroz, 2010; Ko et al., 2008; Horiuchi et al., 2010), are based on wavelet decomposition and sub-band substitution to embed the color information in a gray level image. Their main objectives include compression and image authentication (Campisi et al., 2002; Zhao et al., 2004) and image printing (R. De Queiroz and K. Braun, 2006; R. De Queiroz, 2010; Ko et al., 2008; Horiuchi et al., 2010). Their method is used to recover color information from documents prepared in color but printed with a black-andwhite printer or transmitted by a conventional black-and-white fax machine. Even if those techniques embed the color information, their approach and purpose are clearly different from that explored in this paper. Indeed, none of these techniques try to protect the color information by hiding the color palette within the index image.

Previous work proposed methods for palette reordering in order to improve the compression of color-indexed images (Memon and Venkateswaran, 1996; Pinho and Neves, 2004; Battiato et al., 2007). These approaches show that the palette reordering is very important but depends of the application.

Our previous effort (Chaumont and Puech, 2006, 2007) proposed to protect the color information by hiding the color palette within the index image. In fact, in (Chaumont and Puech, 2006, 2007), an approach is described to reorder the colors of the palette in order to get an index image which is close to the luminance of the original color image, and at the same time a color palette whose consecutive colors are close.

In this paper, in addition to our previous method (Chaumont and Puech, 2006), a way to reduce the number of colors is proposed, while analysing the results as a function of the number of colors for the quantization, and as a function of the size of the layer to build the path. Furthermore, our results are compared with the approach of De Queiroz and Braun (R. De Queiroz and K. Braun, 2006) and that of Campisi et al. (Campisi et al., 2002).

The rest of this paper is organized as follows. Section 2 details the proposed algorithm as applied to color images. In Section 3, the results of the proposed method applied to digi-

seum. Only low quality images are accessible. If the authorized user requires images of better quality, he/she must issue a request to the Louvre Museum. Another protocol is then used to distribute a higher quality image (by CD or with a secure connection). The low quality ensures that the images will not be used for commercial purposes. tal paintings are presented and analysed. Section 4 presents the conclusion.

\section{The proposed method}

After an overview of the method in Section 2.1, the color quantization is detailed in Section 2.2 while Section 2.3 presents the layer scanning algorithm. In Section 2.4, the choice of color number $K \in \mathbb{N}^{*}$ of the palette is explained, in Section 2.5 , the strategy of the data hiding algorithm is presented, and finally in Section 2.6 the security of the approach is discussed.

\subsection{Overview}

The proposed method involves three major steps, which are color quantization, color reordering and the data-hiding algorithm. The overview of the proposed method is presented in Fig. 1.

To quantize the original color image, the luminance image is used in order to choose the number of colors, $K \in \mathbb{N}^{*}$. After quantization, the color reordering algorithm is applied in order to get a reordered color palette and an index image close to the luminance image. The last step consists of embedding the color palette, which is the message, in the index image, which is the cover.

\subsection{Color quantization}

The color quantization method is presented in this section. Reducing the color number of a color image is a classical quantization problem. The optimal solution, to extract the $K$ colors, is obtained by solving equation (1):

$$
\begin{aligned}
\left\{P_{i, k}, C(k)\right\}= & \arg \min _{P_{i, k}, C(k)} \sum_{i=1}^{N} \sum_{k=1}^{K} P_{i, k} \cdot d^{2}(I(i), C(k)) \\
& \text { with } \forall i, \exists ! k^{\prime}, P_{i, k^{\prime}}=1 \text { and } \forall k \neq k^{\prime}, P_{i, k}=0,
\end{aligned}
$$

where $I$ is a color image of dimension $N, C(k)$ is the $k^{\text {th }}$ color of the $K$ searched colors, $d()$ is a distance function in the color space (L2 in the RGB color space), and $P_{i, k} \in\{0,1\}$ is the membership value of pixel $i$ to color $k$. The constraint on $P_{i, k}$ is that for all $i$ there is a single $k$ such that $P_{i, k}=1$. The choice of value of the color number $K$ is presented in detail in Section 2.4.

A well known solution to minimize equation (1), and then to obtain the $K$ colors, is to use the ISODATA k-mean clustering algorithm (Ball and Hall, 1966). $P_{i, k}$ is defined in equation (2):

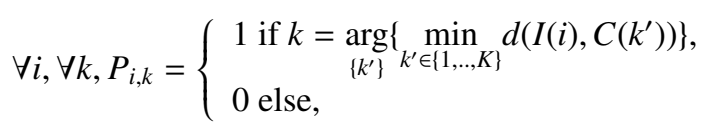

with $C(k)=\frac{\sum_{i=1}^{N} P_{i, k} \times I(i)}{\sum_{i=1}^{N} P_{i, k}}$.

In the approach, the number $K$ is significant in comparison to the original number of colors. With a classical k-mean algorithm, the number of extracted colors will often be below $K$. 


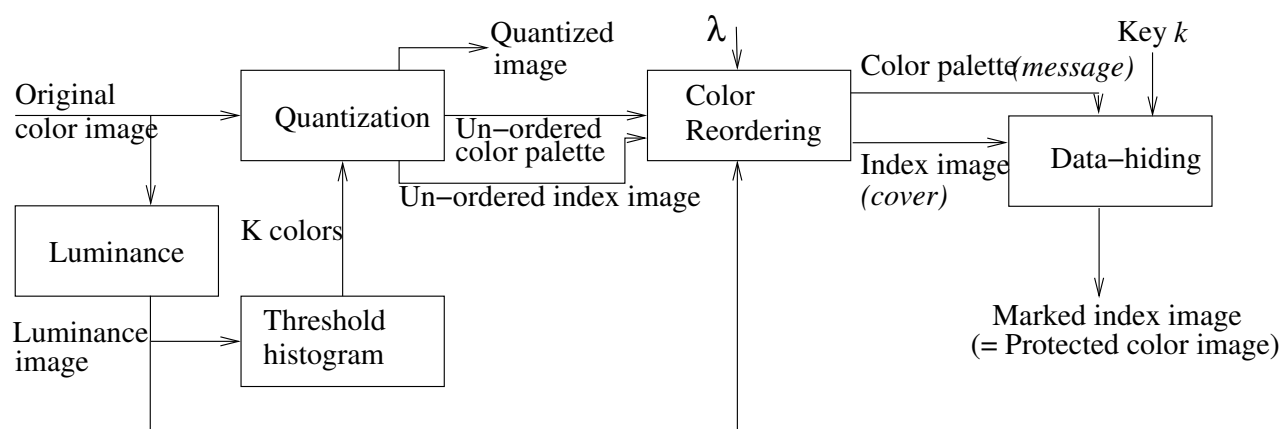

Figure 1: Overview of the method.

Indeed, it is the well known problem of "death classes". To overcome that problem, one could initialize the $P_{i, k}$ values by solving the fuzzy c-mean equation given by equation (3):

$$
\left\{P_{i, k}, C(k)\right\}=\arg \min _{P_{i, k}, C(k)} \sum_{i=1}^{N} \sum_{k=1}^{K} P_{i, k}^{m} \cdot d^{2}(I(i), C(k)),
$$

where $m$ is a fuzzy coefficient (experimentally $m$ is set at 1.6 as proposed in (Castagno and Sodomaco, 1998)) and $P_{i, k}$ are real values in the range $[0,1]$, named fuzzy membership values. This equation is solved by a fuzzy c-mean algorithm (Dunn, 1974).

A quantized image is obtained once the quantization with $K$ colors has been carried out. A color palette and its index image are associated with this quantized image. By applying just the quantization algorithm, the content of the index image does not semantically correspond to the luminance content of the original image. The color palette order should be changed to obtain an index image where its content are semantically close to the luminance content. Consequently the associated index image will change too.

\subsection{Layer scanning algorithm}

In this section, the layer scanning algorithm is presented. After color quantization, the $K$ color image can be represented by an index image (based on $P_{i, k}$ values) and a color palette (based on $C(k)$ values). The index image is denoted Index and is defined such that equation (4):

$$
\forall i \in\{1, . ., N\}, \operatorname{Index}(i)=\arg \max _{k \in\{1, . ., K\}} P_{i, k} .
$$

The color palette is denoted Palette and $\forall k \in$ $\{1, . ., K\}, \operatorname{Palette}(k)=C(k)$.

The goal is then to find a solution by taking two constraints into account. The first constraint is to get an index image where each gray level is close to the luminance of the original color image. The second constraint is that two consecutive colors should be close in the color palette. This second constraint is necessary to preserve a good quality of the reconstructed color image. Thanks to color quantization, we already have an index image and a color palette. The problem is then to find a permutation function that simultaneously permutes the values of the index image and those of the color palette. The best permutation function $\Phi$ is found by solving equation (5):

$$
\Phi=\arg \min _{\Phi}\left[\sum_{i=1}^{N} E_{i}^{\text {ind }}+\lambda \sum_{k=1}^{K-1} E_{k}^{\text {palette }}\right],
$$

where $E_{i}^{i n d}$ (equation(6) is the energy related to the index image and $E_{k}^{\text {palette }}$ (equation(7) the energy related to the color palette:

$$
\begin{gathered}
E_{i}^{\text {ind }}=d^{2}(Y(i), \Phi(\operatorname{Index}(i))), \\
\left.E_{k}^{\text {palette }}=d^{2}\left(\operatorname{Palette}\left(\Phi^{-1}(k)\right), \text { Palette }\left(\Phi^{-1}(k+1)\right)\right)\right],
\end{gathered}
$$

where $Y$ is the luminance of the original color image, and $\lambda \epsilon$ $\mathbb{R}_{+}^{*}$ is a parameter controlling the weight assigned to the two energy terms.

The $\Phi$ permutation function is a bijective function in $\mathbb{N}$ defined by $\Phi:\{1, . ., K\} \rightarrow\{1, . ., K\}$. The minimization of equation (7) is not feasible by using a derivative approach since $\Phi($. and Palette(.) are discrete functions. A metaheuristic approach could be used, such as evolutionist algorithms, for minimizing equation (5), but we prefer a less CPU consuming solution and a less memory costly solution. Equation (5) is thus solved using an heuristic algorithm: the layer scanning algorithm. The aim of this algorithm is to find a reordering of $K$ colors such that consecutive colors are close and the colors are reordered from the darkest to the lightest. This reordering defines, for each $k^{\text {th }}$ color, a $k^{\prime}$ position which gives the $\Phi$ function such that $\Phi(k)=k^{\prime}$.

To reorder the $K$ colors, the algorithm scans the color space to build the reordered suite of colors, as illustrated in Fig. 2. This scanning is obtained by jumping from color to color in the color space, and then choosing the closest color to the current one. The first color of this suite is chosen as the darkest one among the $K$ colors. An additional constraint to this scanning is that the search is restricted to colors which are not too different in terms of luminance. This means that the scanning in the color space is limited to a layer defined on luminance information. This layer scanning algorithm could then be seen as a kind of "3D spiral scan" in the color space. Fig. 2.a, b and c illustrate the progression of this algorithm. Fig. 2.a, shows the selected colors (in black) building the path before the current color (in red). The colors in purple are the candidate colors belonging 


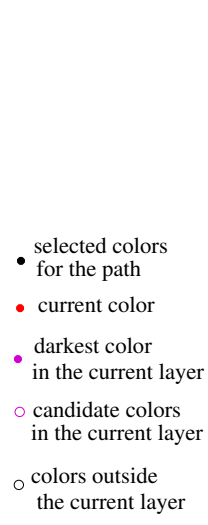

(a)

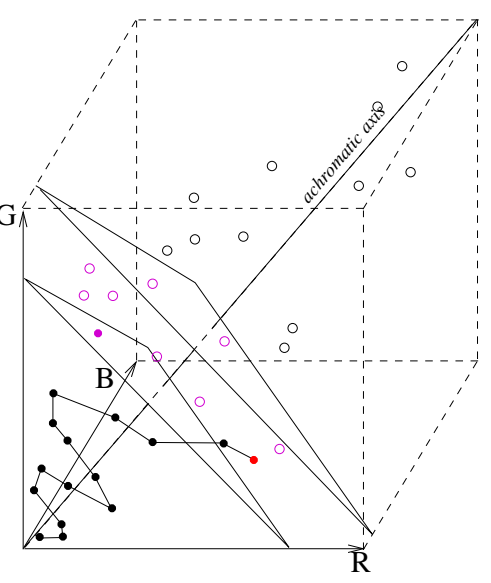

(b)
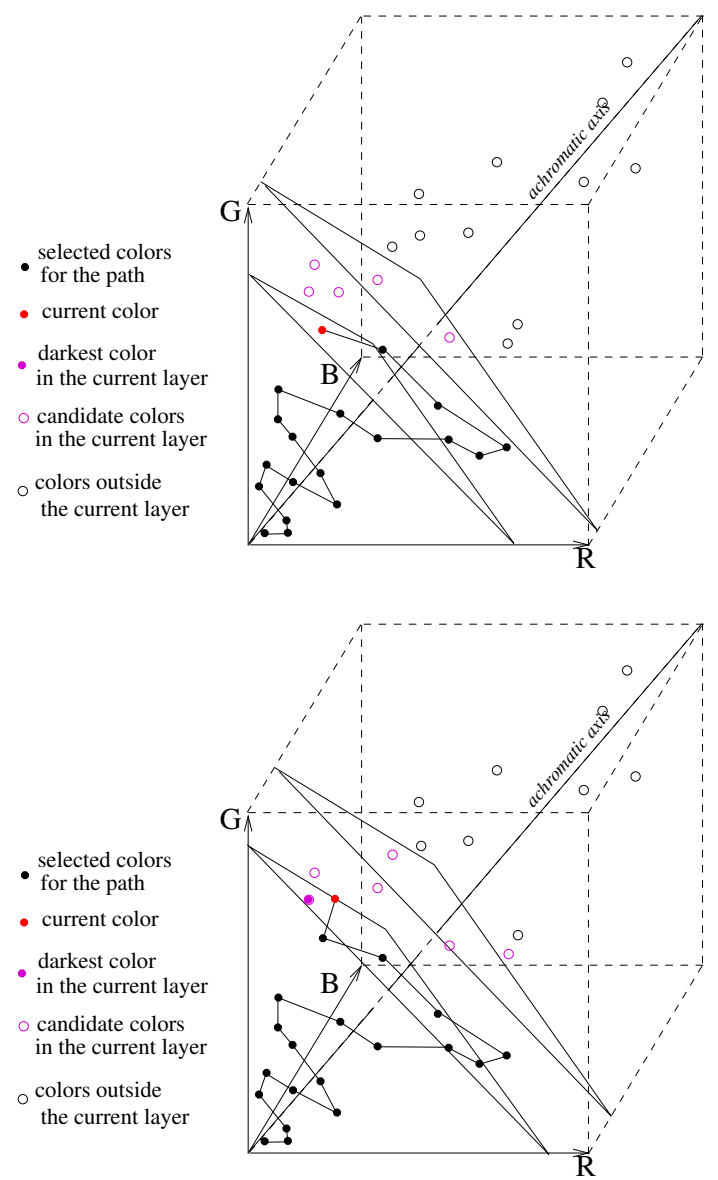

(c)

Figure 2: a) Construction of the path by selection of colors in the current layer, b) Selection of the darkest color of the layer, c) Shifting of the layer until obtaining a new darkest color. to the current layer. One of them (in full purple) is the darkest color of the current layer. In Fig. 2.b, this darkest color of the current layer is selected to build the path. The current layer is thus translated along the achromatic axis in order to adjust the current layer to a new darkest color, as illustrated in Fig. 2.c.

With the application of this layer scanning algorithm, we obtain a reordered color palette and its index image, which is semantically understandable. Note that the application of this algorithm does not change the informational content. Indeed, this new color palette and the associated index image allow for the same color image to be built before and after processing the layer scanning algorithm. The energy shown in equation (6) is related to the index image as a function of the layer size. This energy is minimized when the layer size is small. For example, if the layer size is equal to 1 then the index image is built mainly as a function of the luminance of the palette colors. For the energy related to the color palette, equation (7), the variation as a function of the layer size differs from the previous one. In equation (5), parameter $\lambda$ could give, as a function of its value, more importance to the color palette continuity or to the index image.

This layer running algorithm has an implicit hidden parameter which is the layer size used during the color running in the color space. Since our goal is to minimize the equation (5), a satisfactory way to automatically set this parameter is to test all possible values for this layer size parameter and to keep the layer size value minimizing the equation. Knowing that the possible values of the layer size parameter belong to the range $\{1, \ldots, K\}$ and that it is very fast to make just one run in the color space, this gives an elegant and rapid solution to approximate the equation (5).

Practically, $\lambda$ depends on the parameter $\alpha \in \mathbb{R}_{+}^{*}$ given in equation (8):

$$
\lambda=\alpha \times \frac{N}{K-1},
$$

where $N$ is the image size in pixels. With $\alpha$ set at 1 , the same weight is given to the two constraints. With $\alpha \in[0,1]$, more importance is given to the luminance constraint, and inversely with $\alpha$ greater than 1, more importance is given to the continuity of the color palette. Note that alpha is set by the user.

\subsection{Choice of the color number $K$}

Before explaining the data hiding algorithm in Section 2.5, in this section a solution to improve the quality of the index image is presented as a function of the number of colors, $K$. Because of the quantization step and due to the fact that the pixel size of the index image is 1 byte/pixel, each gray level between 0 and 255 contains several pixels. The index image can then have more contrast than the luminance of the original image. In fact, in the luminance image, fewer gray levels than total are assigned. We could thus decide, from the luminance histogram of the original image, that many gray levels may not be used at all and thus decrease the value of the number $K$ for the quantization step. In the previous section, we explained the method used to build an index image approaching the luminance of the original color image and to have a color palette whose consecutive colors are not too far from each other. The color number 
$K$ was supposed to be known. This section gives an empirical way to choose this color number. One could choose a color number $K$ equal to 256 but it is not adequate to build an index image that is similar to the luminance image. Indeed, the 256 index values are assigned in the index image whereas this is not often the case in the luminance image. A solution is to choose a color number that is equal to the gray level range of the luminance image. Let us modify the energy of equation (5) in order to reduce this color number. Only equation (6) (first term) is substituted by equation (9):

$$
E_{i}^{i n d}=d^{2}(Y(i), t+\Phi(\operatorname{Index}(i))),
$$

where $t$ is a translation value.

The choice of $K$ is guided by the minimization of equation (5), with $E^{\text {ind }}$ the energy related to the Index image, defined by equation (9). Instead of testing all possible values of $K$ and $t$, minimizing equation (5), we adopt an empirical approach. We define a relevance threshold which is defined as a percentage of the maximum histogram value. Gray level values under this threshold are considered negligible. One then defines the relevant gray level interval such that the lower bound is the first relevant gray level index and the upper bound is the last relevant gray level index. The interval size gives the color number $(K)$ and the lower bound gives the translation value $(t)$.

For example, on the Lena image, the maximum value of the histogram is 688 . The relevance threshold is set at $1 \%$ of the maximum value, and is thus equal to 6.88 . By scanning the histogram from values 0 to 256 , the algorithm finds that the gray level 30 is the first relevant level (it is greater than 6.88) and that the gray level 228 is the last relevant level (it is greater than 6.88). The relevant interval is thus $[30,228]$. This interval contains 199 different values; we thus take $K=199$. In order to generate a gray-level image, thanks to the Index image, that has 199 indices, we translate the indices of the Index image with $t=30$. By using this automatic threshold, one can decrease the number of colors $K$ for the quantization step presented in Section 2.2.

Note that giving a color number equal to the relevant interval size reduces the index range, thus giving a less contrasted indexed image. The visual quality of the Index image is then better. The fact of choosing a color number equal to this gray level range ensures a marked reduction in the first energy term (equation (9)), without any substantial growth in the second energy term (equation (7)), and so without any marked distortion in the color image due to data-hiding.

\subsection{Color protection of an image by data-hiding}

In this section, we explain our strategy to embed data of the color palette into the index image. Spatial domain methods embed the information directly into the pixels of the original image. Earlier techniques embedded the bit message in a sequential way in the LSB (Least Significant Bit) of the pixel image (Bender et al., 1996; Nikolaidis and Pitas, 1998). Those were then improved by using a PRNG (Pseudo-Random Number Generator) with a secret key in order to obtain private access to the embedded information. The PRNG spreads the message over the image evenly and makes the steganalysis harder (Fridrich and Goljan, 2002).

For our method, we developed a spatial domain algorithm to embed color palette information in the index image composed of $N$ pixels. The main steps of this data hiding algorithm are the partitioning of the image into blocks of homogeneous size, the selection of one pixel in each block and the substitution of the gray level of each selected pixel by one of its two neighbors of the color palette. We call this index substitution.

The objective of the data hiding step is thus to embed a message $M$ made up of $m$ bits $b_{j}\left(M=b_{1} b_{2} \ldots b_{m}\right)$. The embedding factor, in bit/pixel, is given by equation (10):

$$
E_{f}=m / N \text {. }
$$

The original index image is then partitioned into blocks of size $\left\lfloor 1 / E_{f}\right\rfloor$ pixels. Each block is used to hide only one bit $b_{j}$ of the message. This partition procedure guarantees that the message is spread homogeneously over the whole image. In order to hide the data of the color palette within the image, $m=$ $3 \times K \times 8$ bits must be embedded in the index image. When $K=$ 256 colors (which is the maximal value), the number of bits to embed is $m=6144$ bits and, for an image of $512 \times 512$ pixels, the size of the blocks is 42 pixels and the embedding factor $E_{f}=0.0234$ bit $/$ pixel. Note that the color palette data can be losslessly compressed (Chaumont and Puech, 2007).

In the next step of our data hiding approach, for each block, the PRNG selects a pixel Index(i). If necessary, to get an embedded pixel, Index $x_{W}(i)$, the selected pixel Index $(i)$ is then substituted by one of its two neighbors according to the message bit $b_{j}$ in order to preserve the best quality of the reconstructed image from the data-hidden image, as formalized by equation (11):

$$
\operatorname{Index}_{W}(i)=\left\{\begin{array}{l}
\text { Index }(i) \text { if } b_{j}=\text { Index }(i) \bmod 2, \\
\underset{k \in\{\operatorname{Index}(i)-1, \operatorname{Index}(i)+1\} \cap\{1, \ldots, K\}}{\arg (i)}-\operatorname{Palette}(k))^{2} \text { otherwise }
\end{array}\right.
$$

Thus, the index value $\operatorname{Index}(i)$ is modified by +1 or -1 gray level when $b_{j} \neq \operatorname{Index}(i) \bmod 2$. The best choice for this modification is then to choose the closest color between Palette $(\operatorname{Index}(i)+1)$ and Palette $(\operatorname{Index}(i)-1)$ in order to minimize the distance to the color Palette(Index $(i))$. This way of embedding the color palette ensures that each embedding pixel can be modified only by one gray level and at the same time the reconstructed color pixel from the embedding image is very close to the original color value.

\subsection{Security investigation}

In this section, we investigate the security of our approach. Our approach embeds a specific message (the palette) in an 8bit Index image whose histogram is relatively flat (quasi equiprobability). The embedding process is a +-1 approach and the payload is very small (it is lower than or equal to $3 \times 8 \times$ $256=6144$ bits). For a tiny $256 \times 256$ image, the relative payload is under 0.1 bits per pixel. In the classical framework 
of Kerckoff (Kerckhoffs, 1883), it is assumed that an attacker knows the embedding, the extraction algorithm, and the datahidden image. The only unknown information is the key.

Steganalysis can inform us about the presence of a hidden message and sometimes about the amount of hidden bits. Nevertheless, if the probability distribution of cover images is unknown, steganalysis does not provide information about the positions of the modified pixels (Fridrich, 2009). Note that the best efficient attack, on a +-1 embedding algorithm, on natural images that have been quantized, is only able to guess if there is or not a hidden message (and the probability of error is often very high at low payload) (Kodovský and Fridrich, 2012). The index images (from palette based approaches) are similar to quantized images in the sense that there is a reduction in the number of different values. Note also that the best attack, with a LSB embedding algorithm (+-1 embedding is more secure), with palette based images, is only able to estimate the number of embedded bits (Pairs Analysis attack (Fridrich, 2009)) and not the localization. It is nowadays impossible to estimate the positions where a modification has occurred for +-1 approaches (see for example the paper (Quach, 2012)). Even if we were able to guess the pixels that have been modified, it is even more difficult to guess the pixels that have not been modified and that hide one bit. That said, we do not propose a steganographic scheme and do not seek to create a secret communication channel. We only want to make the message inaccessible. In this, the steganalysis does not endanger our system of concealment.

On the other hand, we do not attempt to have a message that will survive image degradation. We are not looking for a robust watermarking scheme; we are not trying to create a reliable communication channel. In addition, knowing that the message (the palette) varies for each picture, attacks on security based on the observation of many watermarked images are unusable (Cayre et al., 2005).

Finally, assuming that the embedded sequence is extracted, an attacker would have to reorder it. Indeed, the embedding is achieved through a pseudo-random path in the Index image. An attacker will have to break the PRNG in order to retrieve the right path, and this is a difficult task.

In order to finalize this security analysis, we must talk of the colorization attack (Chaumont and Puech, 2008). The colorization attack allows building of a color image from an Index image that hides the palette. It is an indirect attack similar in spirit to a protocol attack. The obtained image is not colorimetrically identical to the original image. The attack necessitates a human intervention, and thus the attack does not work on images whose interpretation by a human is difficult. That kind of attack implies a possible weakness in the scheme, and this should be considered in the future in order to increase the security of the scheme.

\section{Experimental results}

We applied our method to several digital paintings of the EROS database of the C2RMF laboratory ${ }^{2}$, near the Louvre

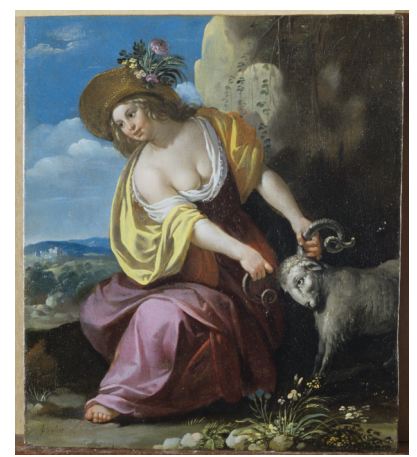

(a)

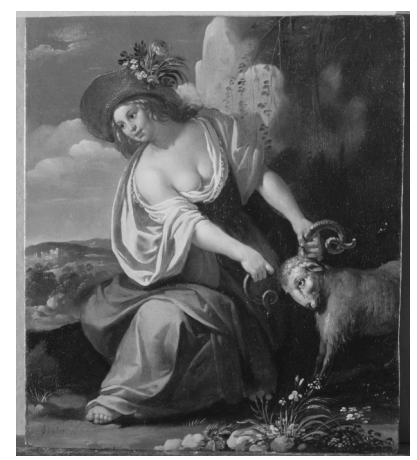

(b)

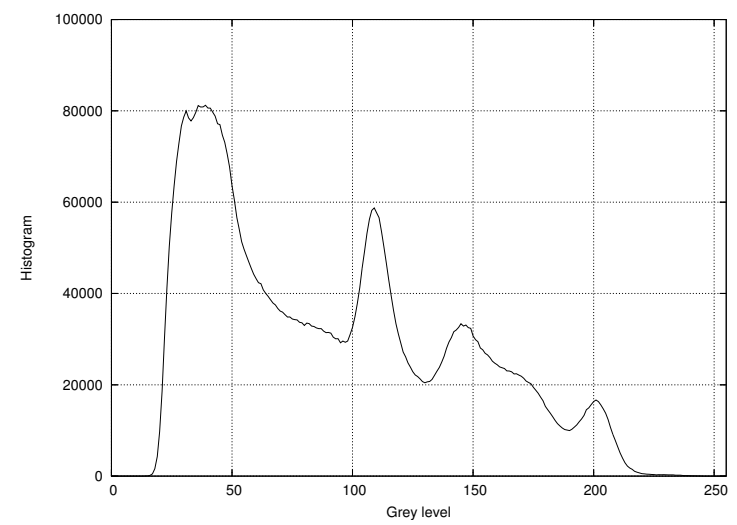

(c)

Figure 3: a) Original color image $(2450 \times 2763$ pixels $)$ : Woman with a ram by Jan van Bylert, oil on oak, conserved at the Saint Germain-en-Laye city Museum, (inv. 872.1.22), b) Luminance of the original color image, c) Histogram of the luminance.

\footnotetext{
${ }^{2}$ Centre de Recherche et de Restauration des Musés de France, Paris.
} 
Museum, Paris. To illustrate our approach, we present the results of our method applied to two digital paintings. The first digital painting is the Woman with a ram by Jan van Bylert, illustrated in Fig. 3.a, and the second one is the famous portrait of Mona Lisa by Leonardo da Vinci, as given in Fig. 18.a. Note that the computational complexity of our approach is similar to approaches based on wavelet substitution. On a low cost laptop, with an Intel(R) Core TM2 Duo CPU 8600 processor running at $2.4 \mathrm{GHz}$ with $4 \mathrm{~GB}$ RAM, the longest execution time, over 5 trials, on the $256 \times 256$ pixels Lena image, is 0.98 second for our approach ${ }^{3}, 0.13$ second for Campisi et al. approach (Campisi et al., 2002), and 0.1 second for De Queiroz and Braun approach (R. De Queiroz and K. Braun, 2006).

\subsection{A full example on a digital painting \\ 3.1.1. Application of the layer scanning algorithm}

The first $2450 \times 2763$ digital painting, illustrated in Fig. 3.a, is from the original oil on oak painting by Jan van Bylert, called the Woman with a ram. The original is conserved at the Saint Germain-en-Laye city Museum, (inv. 872.1.22). The luminance image of this digital painting is shown in Fig. 3.b and its histogram in Fig. 3.c.

Quantization with $K=256$ colors results in the quantized image given in Fig. 4.a. The difference between the original image (Fig. 3.a) and the quantized one (Fig. 4.a) gives a color $P S N R=37.17 d B$, which implies a good quality. Associated with this quantized image, is a color palette and its index image, as illustrated in Fig. 4.b, and Fig. 4.c. We can observe that the content of the index image does not semantically correspond to the luminance content of the original image. To have an index image with its content semantically close to that of the luminance content, we should change the order of the palette colors. Consequently, the associated index image will change too.

With the application of the layer scanning algorithm described in Section 2.3, we obtain the reordered color palette given in Fig. 5.a and its index image (Fig. 5.b) which is semantically intelligible. Note that the layer scanning algorithm does not change the informational content. Indeed, this new color palette and the associated index image allow the same color image to be built before and after processing the layer scanning algorithm. The difference between the luminance of the original image (Fig. 3.b) and the new index image (Fig. 5.b) gives a $P S N R=18.31 \mathrm{~dB}$. Even though this PSNR value is not very high, and the histograms in Fig. 3.c and Fig. 5.c are different, the content of Fig. 5.b strongly resembles the luminance of the original image. The path built in the RGB space with the layer scanning algorithm is illustrated in Fig. 6.

The energy (equation (6)) related to the index image is graphically presented in Fig. 7.a as a function of the layer size. Note that this energy is minimized when the layer size is small. Indeed, when the layer size is unity, then the index image is built mainly as a function of the luminance of the color palette. For the energy related to the color palette (equation (7)), the variation as a function of the layer size (Fig. 7.b) differs from the

\footnotetext{
${ }^{3}$ In that test, the color quantization is achieved with the fast octree quantization algorithm (Gervautz and Purgathofer, 1990).
}

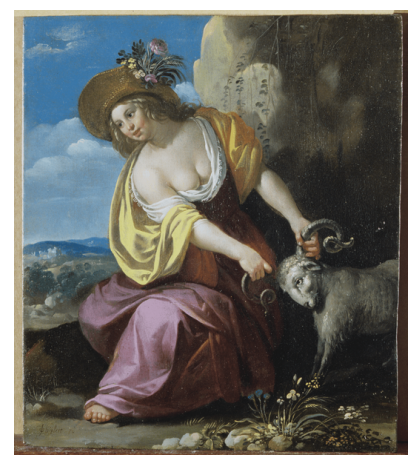

(a)

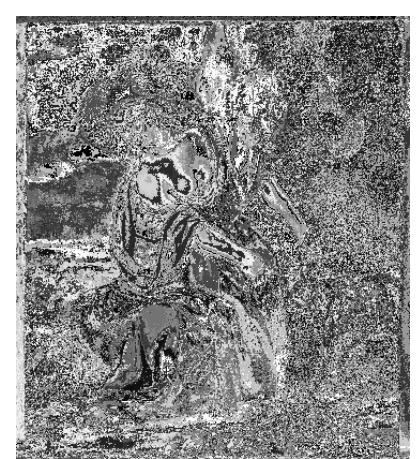

(b)

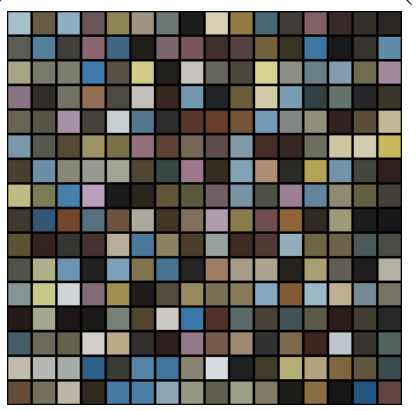

(c)

Figure 4: a) Quantized image with $K=256$ colors, c) Index image from the quantized image (a), b) Color palette of the quantized image (a).

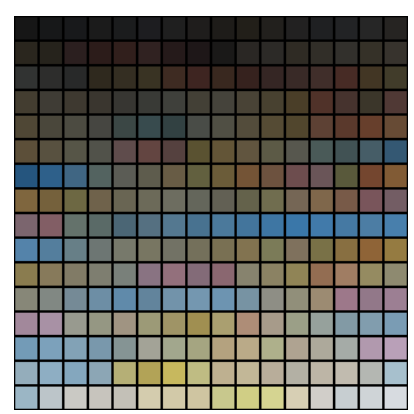

(a)

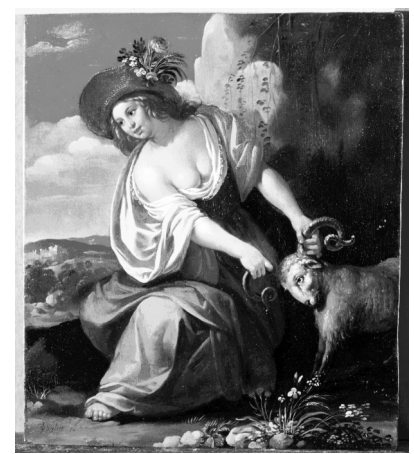

(b)

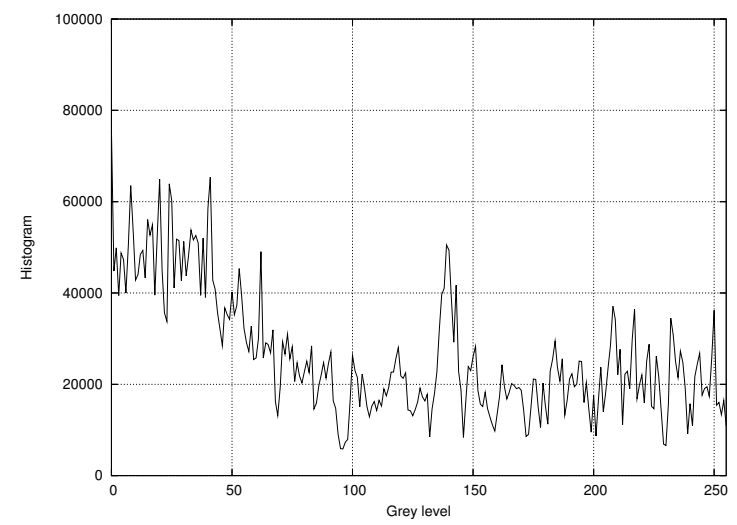

(c)

Figure 5: Application of the layer scanning algorithm (with $K=256$ colors): a) Color Palette after the color reordering, b) Index image after the color reordering, c) Histogram of the index image (b). 


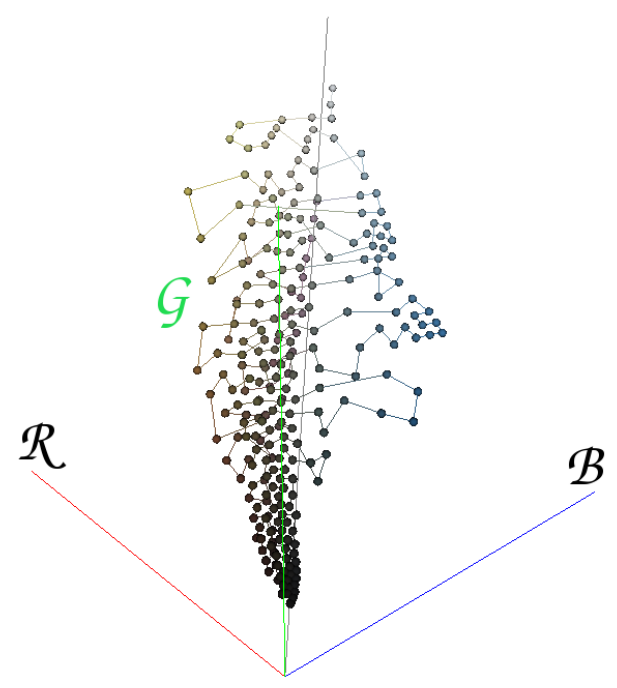

Figure 6: A view of the path built with the layer scanning algorithm in the RGB space.

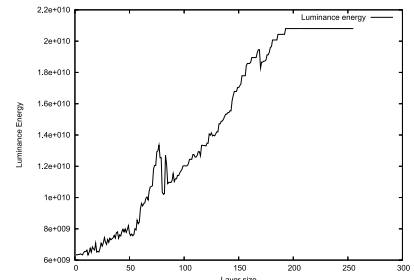

(a)

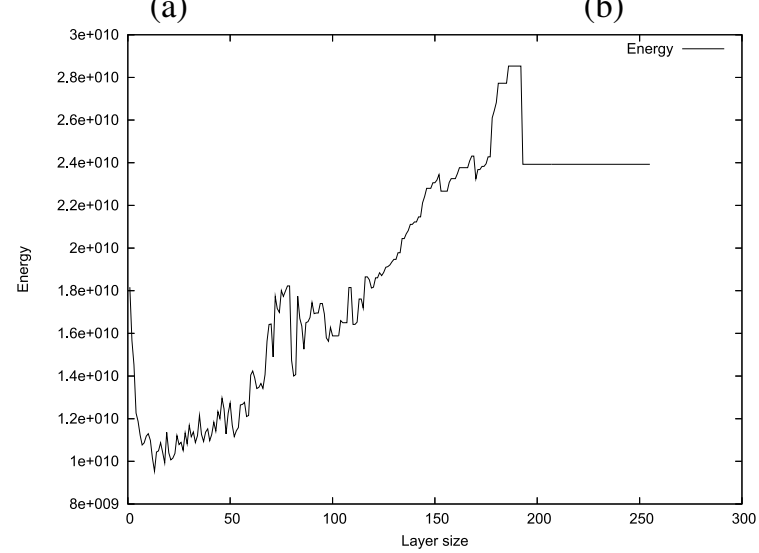

(c)

Figure 7: Energy as a function of the value of the layer (for $K=256$ colors): a) Energy of the luminance, b) Energy of the color palette, c) Total energy with $\alpha=5$. previous one. The lowest energy is observed when the layer size is 20 . In equation (5), the $\lambda$ value could give, as a function of its value, more importance to the color palette continuity or more importance to the index image. We have seen in equation (8) that this $\lambda$ value depends on the $\alpha$ value. To have the best color palette continuity, with $\alpha=5$, we have the minimum value of the total energy for a layer size of 20, as illustrated in Fig. 7.c.

\subsubsection{Choice of the number of colors}

By analyzing the histogram of luminance (Fig. 3), one can deduce that the choice of the color number $K$ can be modified to improve the quality of the index image. With an automatic threshold, set at $1 \%$ of the maximum histogram value, as explained in Section 2.4, we get a relevant gray level interval of $[19,208]$ for our example. We can thus automatically deduce a color number $K=189$ and a translation value $t=19$ in equation (9). Fig. 8 illustrates the results with this new number of colors. With $K=189$, the difference between the original image (Fig. 3.a) and the quantized one (Fig. 8.a) gives a color PSNR of $35.95 d B$, which still corresponds to a good quality. The difference between the luminance of the original image (Fig. 3.b) and the new index image (Fig. 8.c) gives a PSNR of $19.64 d B$. A visual comparison between Fig. 3.b and Fig. 8.c reveals a more pleasant (less contrasted) gray level image with $K=189$ colors. For the quantized images (Fig. 3.a and 8.a), no marked visual difference could be noticed between $K=189$ colors and $K=255$ colors.

Choosing a color number equal to the gray level range ensures a marked reduction in the first term of the energy equation (9) without any substantial growth in the second energy term of the equation (7), and thus without any significant growth in the distortion on the color image due to data-hiding. In Fig. 9, the minimum total energy is obtained for a layer size of 20 (with $\alpha=5$ ).

\subsubsection{Analysis of the layer size}

The optimal layer size value changes if we change the value of $\alpha$. Fig. 10.a and b present the total energy for $\alpha=0$ and for $\alpha=1000$ respectively. The optimal values for the layer size are equal to 3 for $\alpha=0$ and 166 for $\alpha=1000$. The quantized image does not change even if the $\alpha$ value and of the layer size are changed. Of course, we do not change the colors of the palette. On the other hand, the color order changes in the palette and the index images are different.

We have seen that with $\alpha=5$ the optimal layer size is 20 and the difference between the luminance and the index image gives a PSNR of $19.64 d B$. With $\alpha=0$, the layer optimal size is 3 and the PSNR between the luminance and the index image is $19.95 d B$. In this case, the quality of the index image is slightly better than with $\alpha=5$, as illustrated Fig. 11.b. With $\alpha=1000$, the optimal layer size is 166 and the difference between the luminance and the index image gives a PSNR of $12.16 \mathrm{~dB}$. In this case, the quality index image (Fig. 12.a) is bad. For all three values of $\alpha$, the PSNR of the difference between the original image and the quantized one is still $35.95 \mathrm{~dB}$. 


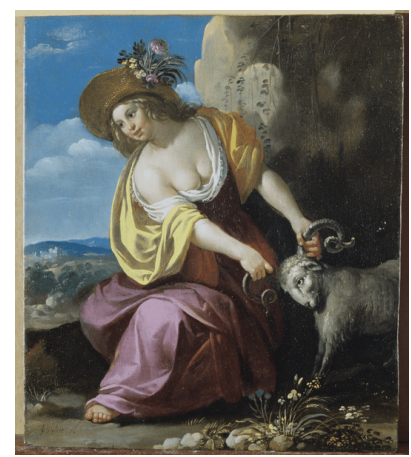

(a)

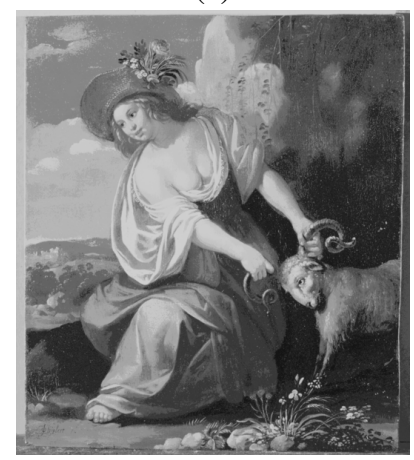

(c)

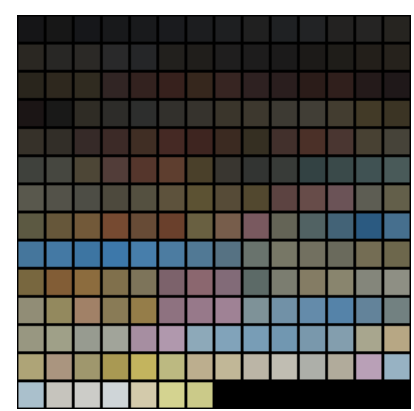

(b)

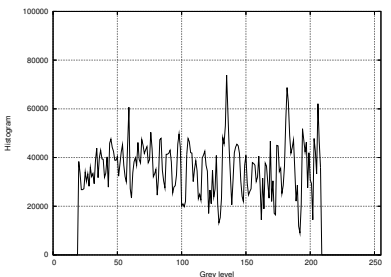

(d)
Figure 8: Application of the layer scanning algorithm with $K=189$ colors and $t=19$ : a) Quantized image, b) Color Palette after the color reordering, c) Index image after the color reordering, d) Histogram of the index image (b).

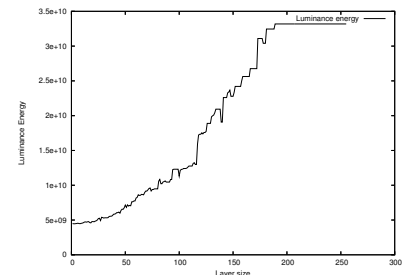

(a)

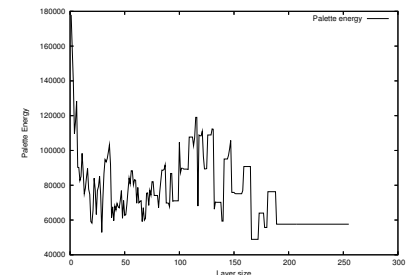

(b)

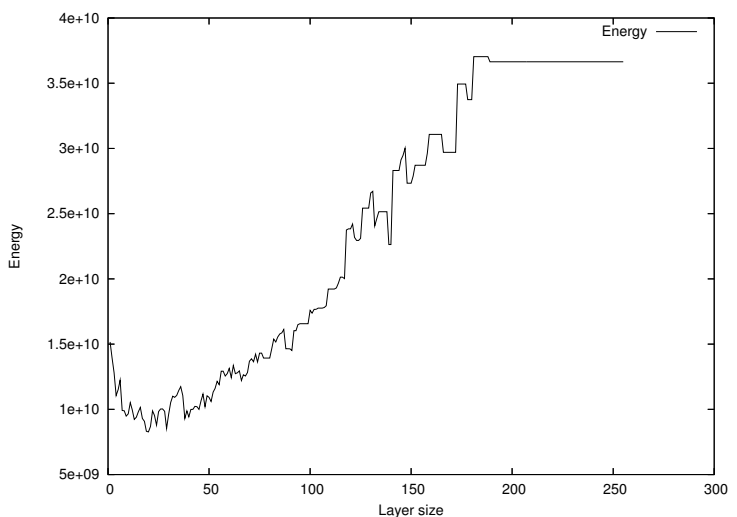

(c)

Figure 9: Energy as a function of the value of the layer (for $K=189$ colors): a) Energy of the luminance, b) Energy of the color palette, c) Total energy with $\alpha=5$.

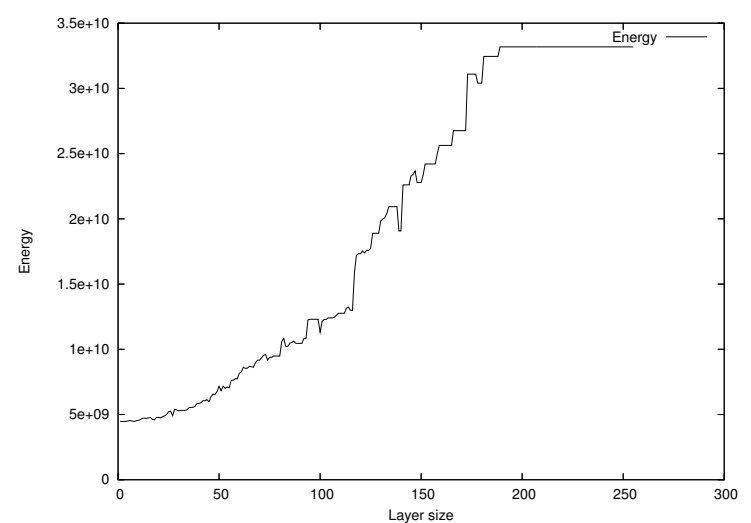

(a)

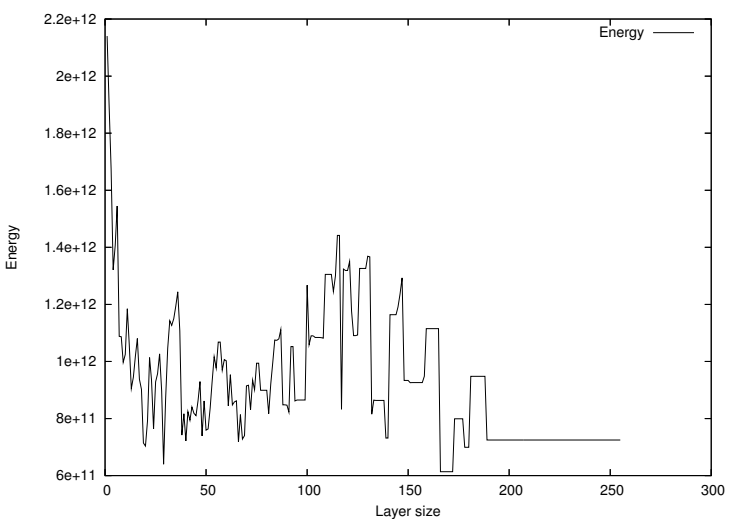

(b)

Figure 10: Total energy as a function of the value $\alpha$ (for $K=189$ colors): a) Total energy with $\alpha=0$, b) Total energy with $\alpha=1000$.

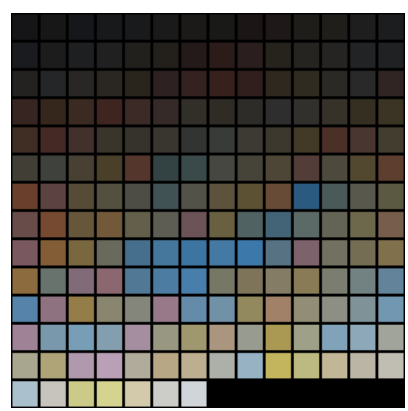

(a)

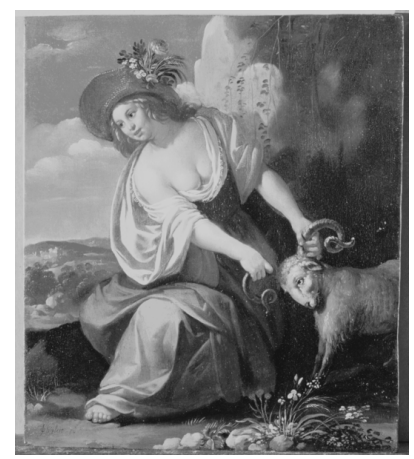

(b)
Figure 11: Application of the layer scanning algorithm with $K=189, t=19$ and $\alpha=0$ (layer size $=3$ ): a) Color palette, b) Index image. 


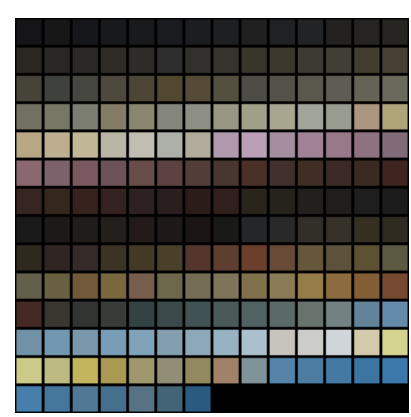

(a)

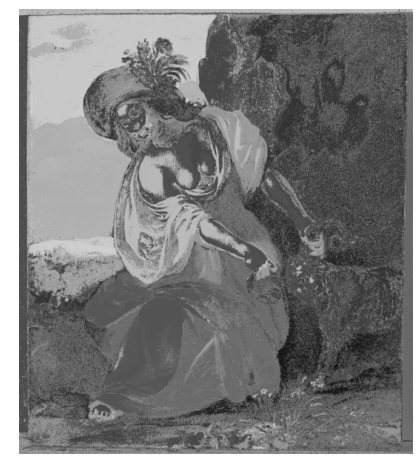

(b)

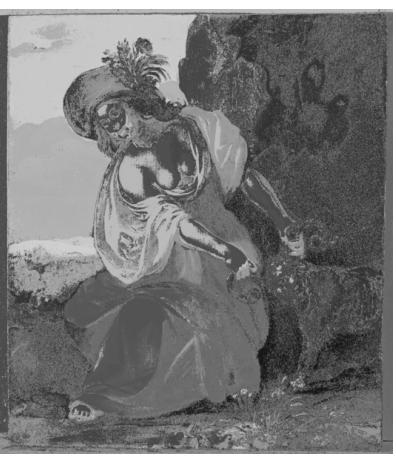

(a)

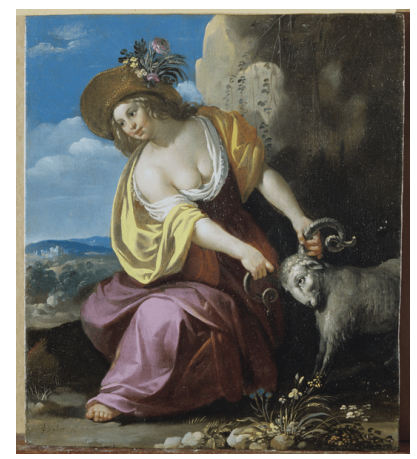

(b)

Figure 12: Application of the layer scanning algorithm with $K=189, t=19$ and $\alpha=1000$ (layer size $=166$ ): a) Color palette, b) Index image.

\subsubsection{Data hiding step}

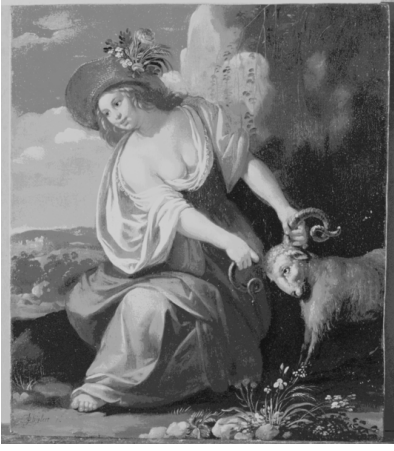

(a)

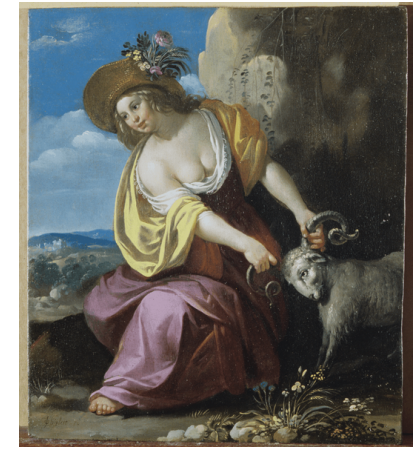

(b)
Figure 13: a) Marked index image of the Fig. 8.c with $K=189, t=19$ and $\alpha=5$ (layer size $=20$ ), b) Reconstructed color image from the marked index image.

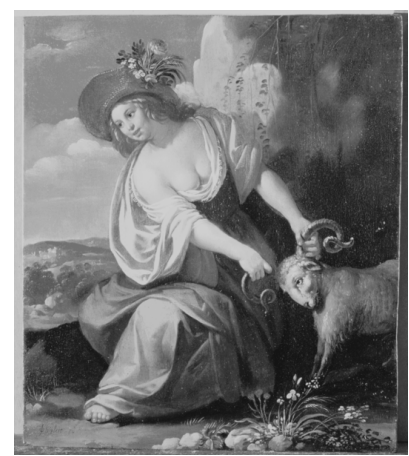

(a)

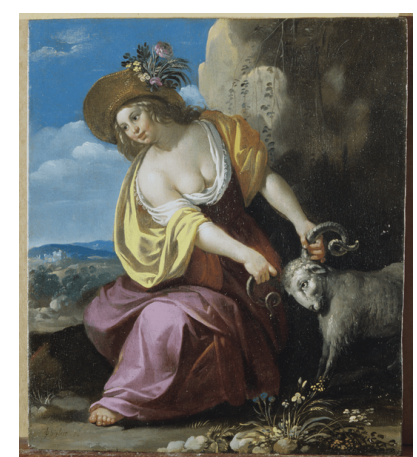

(b)
Figure 14: a) Marked index image of the Fig. 11.c with $K=189, t=19$ and $\alpha=0$ (layer size $=3$ ), b) Reconstructed color image from the marked index image.

In Fig. 13.a $(\alpha=5)$, with the data hiding algorithm presented in Section 2.5, we have embedded the color palette of 189 colors in the range [20,208]. The size of the data is 4536
Figure 15: a) Marked index image of the Fig. 11.c with $K=189, t=19$ and $\alpha=1000$ (layer size $=166$ ), b) Reconstructed color image from the marked index image.

\begin{tabular}{|c|c||c|c||c|c|}
\hline$\alpha$ & $\begin{array}{c}\text { layer } \\
\text { size }\end{array}$ & $\begin{array}{c}\text { PSNR } \\
\text { luminance } \\
\text { index }\end{array}$ & $\begin{array}{c}\text { PSNR } \\
\text { luminance } \\
\text { marked index }\end{array}$ & $\begin{array}{c}\text { PSNR } \\
\text { quantized } \\
\text { rebuilt }\end{array}$ & $\begin{array}{c}\text { PSNR } \\
\text { original } \\
\text { rebuilt }\end{array}$ \\
\hline \hline 0 & 3 & $19.95 \mathrm{~dB}$ & $19.95 \mathrm{~dB}$ & $63.51 \mathrm{~dB}$ & $35.95 \mathrm{~dB}$ \\
\hline 5 & 20 & $19.64 \mathrm{~dB}$ & $19.64 \mathrm{~dB}$ & $65.81 \mathrm{~dB}$ & $35.95 \mathrm{~dB}$ \\
\hline 1000 & 166 & $12.16 \mathrm{~dB}$ & $12.16 \mathrm{~dB}$ & $66.22 \mathrm{~dB}$ & $35.95 \mathrm{~dB}$ \\
\hline
\end{tabular}

Table 1: Results of our approach with 189 colors for 3 values of $\alpha$.

bits. Between the index image (Fig. 8.c) and the marked index image (Fig.13.a), a PSNR of $82.99 d B$ is obtained. This value is very high because the embedding factor is very small; i.e. $E_{f}=6.710^{-4} \mathrm{bit} /$ pixel. The PSNR between the original luminance and the marked index image is $19.64 d B$. Fig. 13.b shows the reconstructed color image from the index marked image. This image is visually close to the original quantized image and the PSNR between the original image and the reconstructed image is $35.95 \mathrm{~dB}$, which confirms this feeling.

If we change the value of $\alpha$ and thus the layer size, we get for $\alpha=0$, the results illustrated in Fig. 14 and, for $\alpha=1000$, the results illustrated in Fig. 15. The PSNR details are presented in Table 1. Because of the small embedding factor, the data hiding step clearly does not change the quality of the index images, whatever the value of $\alpha$. Secondly, one can verify that the best quality for the marked index image is at $\alpha=0$ and the best quality for the reconstructed image is at $\alpha=1000$ when comparing the quantized images with the reconstructed images. Lastly, the qualities of the reconstructed images compared to the original one are the same irrespective of the value of $\alpha$.

\subsection{Comparisons to the state-of-the-art}

The approaches of De Queiroz and Braun (R. De Queiroz and K. Braun, 2006) and Campisi et al. (Campisi et al., 2002) apply a wavelet transform on the luminance and replace some subbands by the two sub-sampled planes of chrominance. The major difference between these two approaches is in the choice of substituted sub-bands. Figures 16.c and 17.c show the wavelet 
decomposition of the luminance of the Woman with a ram image after substitution of some sub-bands by chroma planes.

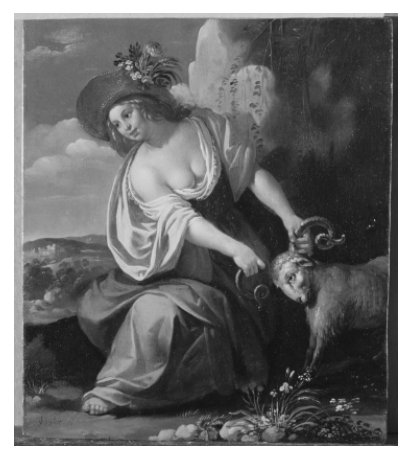

(a)

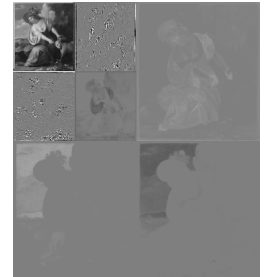

(c)

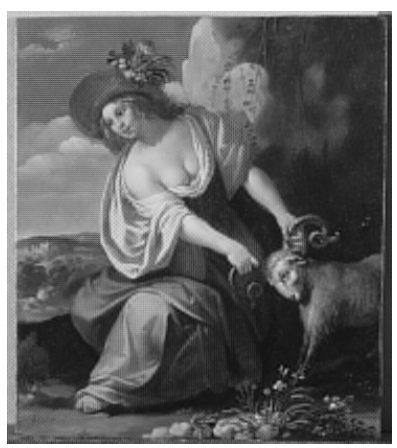

(b)

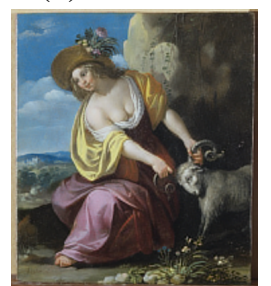

(e)

Figure 16: Application of the substitution-based approach of (R. De Queiroz and K. Braun, 2006): a) Luminance of t he original color image, b) Greylevel image embedding the chrominance planes $(\mathrm{PSNR}=28.01 \mathrm{~dB}), \mathrm{c})$ Wavelet decomposition of the grey-level image, d) Original color image, e) Rebuilt color image from the grey-level image $(\mathrm{PSNR}=30.837 \mathrm{~dB})$.

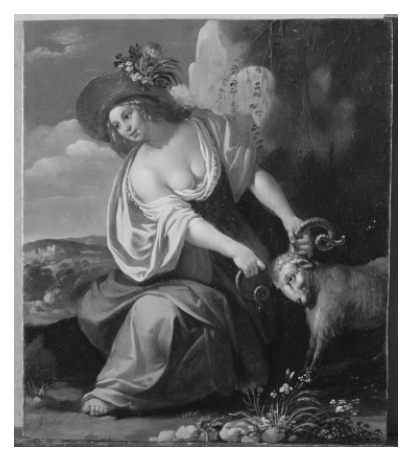

(a)

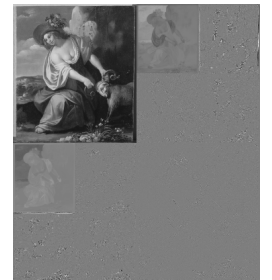

(c)

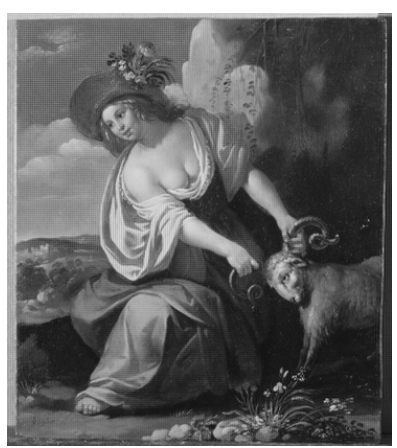

(b)

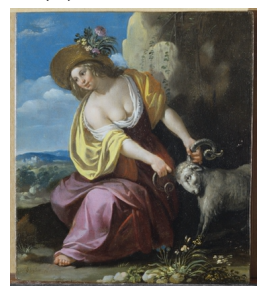

(e)
Figure 17: Application of the substitution-based approach of (Campisi et al., 2002): a) Luminance of the original color image, b) Grey-level image embedding the chrominance planes $(\mathrm{PSNR}=33.94 \mathrm{~dB})$, c) Wavelet decomposition of the grey-level image, d) Original color image, e) Rebuilt color image from the grey-level image $($ PSNR $=34.77 \mathrm{~dB})$.

Usually the color PSNRs of the Campisi et al. approach are better than those of De Queiroz and Braun. In the Woman with a ram image, resized to $368 \times 414$, the color PSNR is 30.84 $\mathrm{dB}$ for the De Queiroz and Braun approach and $34.77 \mathrm{~dB}$ for the Campisi et al. approach. As a reminder, our approach with 256 colors provides a PSNR of $37.17 \mathrm{~dB}$, and with 189 colors provides a PSNR of $35.95 \mathrm{~dB}$. In the Woman with a ram image, $2450 \times 2763$, we get a PSNR of $37.13 \mathrm{~dB}$ for the De Queiroz and Braun approach and a PSNR of $36.64 \mathrm{~dB}$ for the Campisi et al. approach.

On very large sized images, the power of high frequencies is much lower and their destruction, due to the substitution of subbands, is much less harmful to the reconstructed color image. In this case, the two approaches have similar performance to the palette approach with 256 colors and slightly better than the palette approach with 189 colors. Note also that the PSNR range for the palette approach can be easily improved by using, for example, octree quantization followed by a c-mean of 100 iterations as proposed in (Chaumont and Puech, 2007). In this case, the PSNR for the Woman with a ram image, with 256 colors, is greater than $38.8 \mathrm{~dB}$. This again gives slightly higher performance for the palette approach than the substitution of sub-bands approach.

In Figure 18, the results of the different approaches on the Mona Lisa painting are shown. The Mona Lisa image (Fig. 18.a, $925 \times 1382$ pixels), concerns an oil on poplar painting by Leonardo da Vinci (1503-1506) which is conserved at the Louvre Museum (inv 779, copyright C2RMF/Philippe Colantoni). The color PSNRs for the palette approach with 256 colors, the palette approach with 208 colors, the approach of De Queiroz and Braun, and the approach of Campisi et al.. are respectively $38.31 \mathrm{~dB}, 37.73 \mathrm{~dB}, 28.81 \mathrm{~dB}$ and $31.22 \mathrm{~dB}$. Table 2 also gives the PSNR results on well-known images. The palette approach with a color number of less than 256, gives rebuilt color images of PSNR greater than $1 \mathrm{~dB}$ up to $6 \mathrm{~dB}$ compared to the approach of Campisi et al. (Campisi et al., 2002), and greater than $7 \mathrm{~dB}$ up to $10 \mathrm{~dB}$ compared to the approach of De Queiroz and Braun (R. De Queiroz and K. Braun, 2006).

Table 2: PSNR comparisons with well-known images.

\begin{tabular}{|cl|c|c|c|c|}
\hline Images & $\begin{array}{c}\text { De Queiroz } \\
\text { and Braun }\end{array}$ & $\begin{array}{c}\text { Campisi } \\
\text { et al. }\end{array}$ & $\begin{array}{c}\text { Palette } \\
256 \text { colors }\end{array}$ & $\begin{array}{c}\text { Palette } \\
<256 \text { colors }\end{array}$ \\
\hline baboon & PSNR $_{\text {grey }}$ & $21.04 \mathrm{~dB}$ & $27.37 \mathrm{~dB}$ & $16.75 \mathrm{~dB}$ & $18.50 \mathrm{~dB}$ \\
$256 \times 256$ & PSNR $_{\text {color }}$ & $23.93 \mathrm{~dB}$ & $29.8 \mathrm{~dB}$ & $33.31 \mathrm{~dB}$ & $32.58 \mathrm{~dB}$ \\
\hline barbara & PSNR $_{\text {grey }}$ & $23.93 \mathrm{~dB}$ & $30.61 \mathrm{~dB}$ & $18.56 \mathrm{~dB}$ & $21.8 \mathrm{~dB}$ \\
$315 \times 230$ & PSNR $_{\text {color }}$ & $26.33 \mathrm{~dB}$ & $31.75 \mathrm{~dB}$ & $35.95 \mathrm{~dB}$ & $35.75 \mathrm{~dB}$ \\
\hline peppers & PSNR $_{\text {grey }}$ & $21.24 \mathrm{~dB}$ & $25.82 \mathrm{~dB}$ & $19.76 \mathrm{~dB}$ & $25.41 \mathrm{~dB}$ \\
$256 \times 256$ & PSNR $_{\text {color }}$ & $28.82 \mathrm{~dB}$ & $32.36 \mathrm{~dB}$ & $36.32 \mathrm{~dB}$ & $35.76 \mathrm{~dB}$ \\
\hline lena & PSNR $_{\text {grey }}$ & $21.02 \mathrm{~dB}$ & $26.91 \mathrm{~dB}$ & $19.11 \mathrm{~dB}$ & $25.33 \mathrm{~dB}$ \\
$315 \times 230$ & PSNR $_{\text {color }}$ & $30.31 \mathrm{~dB}$ & $36.75 \mathrm{~dB}$ & $38.63 \mathrm{~dB}$ & $37.88 \mathrm{~dB}$ \\
\hline house & PSNR $_{\text {grey }}$ & $25.18 \mathrm{~dB}$ & $30.76 \mathrm{~dB}$ & $18.64 \mathrm{~dB}$ & $23.62 \mathrm{~dB}$ \\
$256 \times 256$ & PSNR $_{\text {color }}$ & $30.75 \mathrm{~dB}$ & $31.76 \mathrm{~dB}$ & $39.27 \mathrm{~dB}$ & $37.95 \mathrm{~dB}$ \\
\hline airplane & PSNR $_{\text {grey }}$ & $26.47 \mathrm{~dB}$ & $34.12 \mathrm{~dB}$ & $12.87 \mathrm{~dB}$ & $13.52 \mathrm{~dB}$ \\
$256 \times 256$ & PSNR $_{\text {color }}$ & $28.82 \mathrm{~dB}$ & $32.58 \mathrm{~dB}$ & $39.90 \mathrm{~dB}$ & $39.04 \mathrm{~dB}$ \\
\hline
\end{tabular}




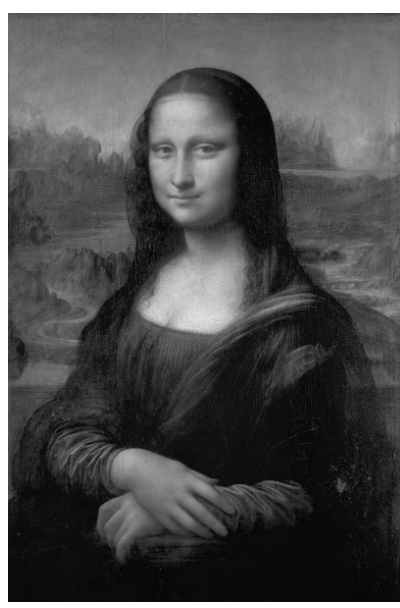

(a) Luminance

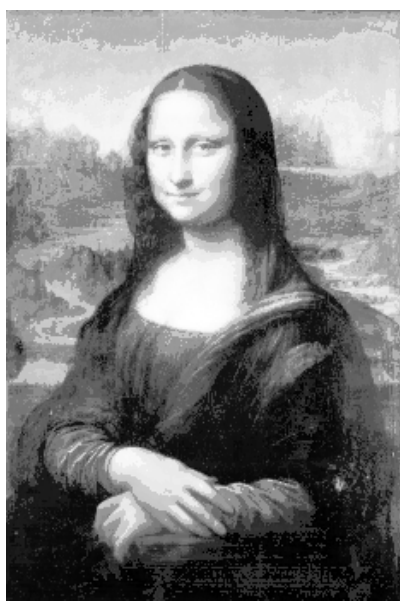

(c) Marked $\mathrm{K}=256$

PSNR $=11.91 \mathrm{~dB}$

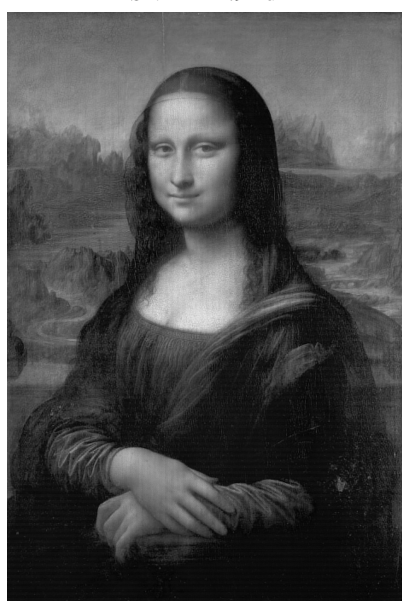

(g) Queiroz \& Braun $\mathrm{PSNR}=26.52 \mathrm{~dB}$

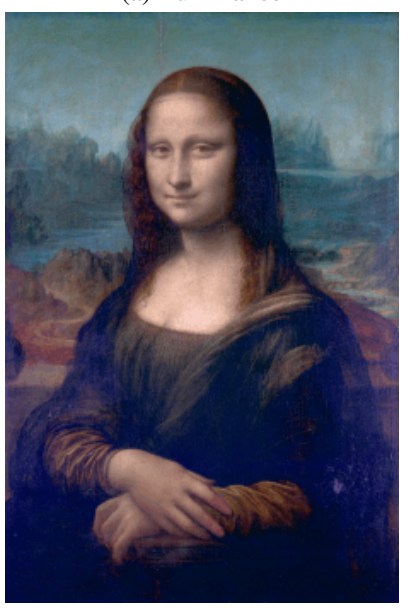

(d) Rebuilt from (c)

PSNR $=38.31 \mathrm{~dB}$

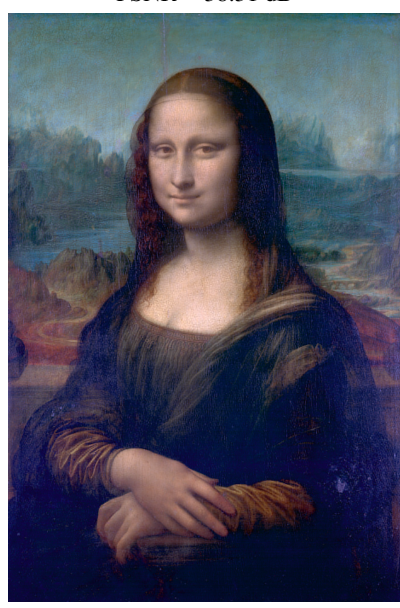

(h) Rebuilt from (g)

PSNR $=28.81 \mathrm{~dB}$

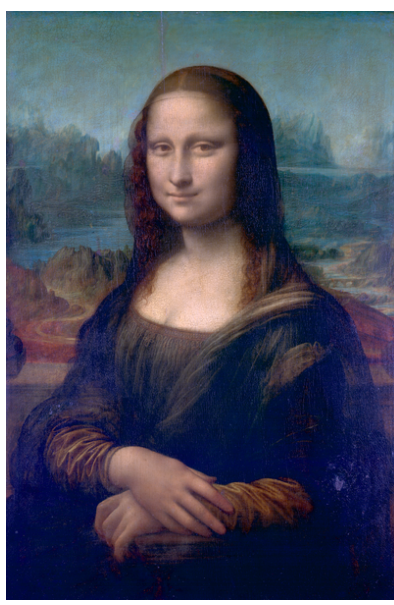

(b) Color

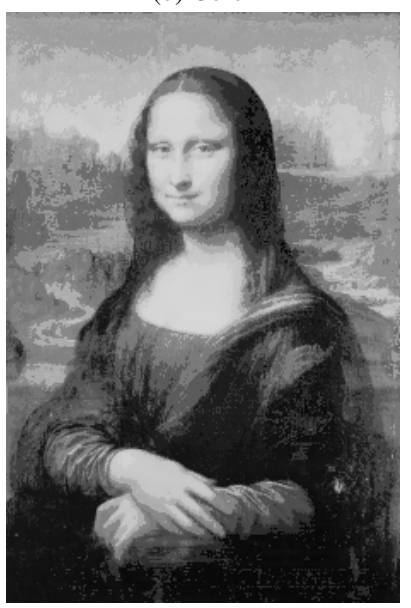

(e) Marked K=208

PSNR $=15.38 \mathrm{~dB}$

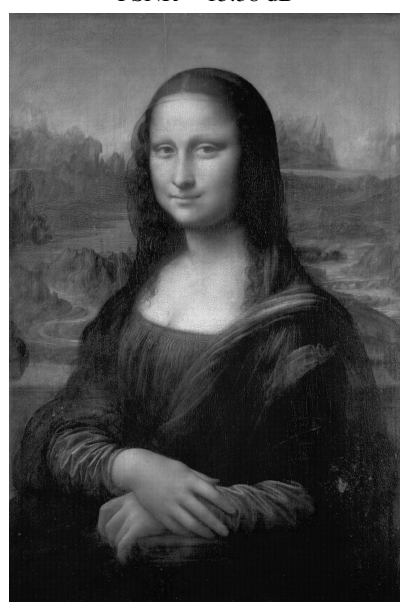

(i) Campisi et al.

PSNR $=31.68 \mathrm{~dB}$

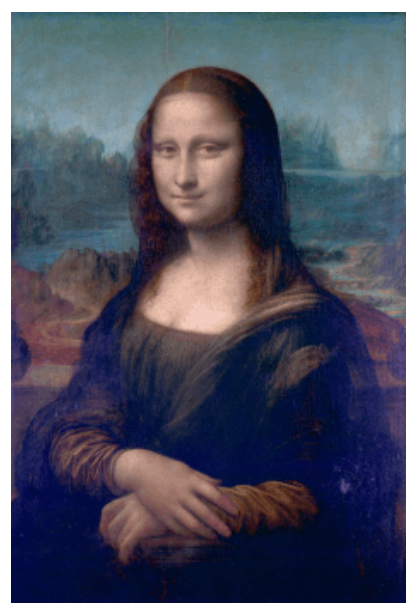

(f) Rebuilt from (e)

PSNR $=37.73 \mathrm{~dB}$

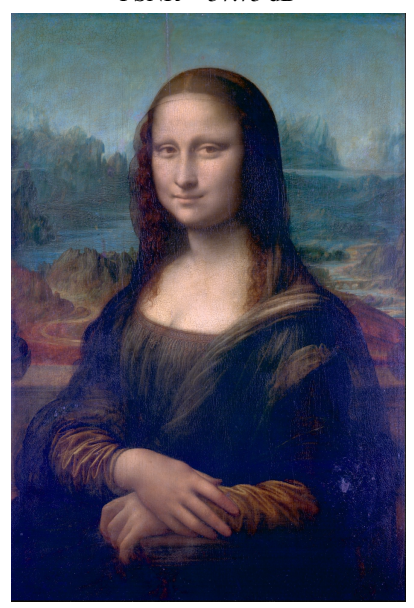

(j) Rebuilt from (i)

PSNR $=31.22 \mathrm{~dB}$

Figure 18: a) Luminance of the original Mona Lisa color image, b) Mona Lisa color image, c) Marked index image $(K=256, \alpha=5$, layer size $=20)$, d) Reconstructed color image from image (c), e) Marked index image ( $K=208, t=8, \alpha=5$, layer size $=20)$, f) Reconstructed color image from image (e), g) Grey-level image with the De Queiroz and Braun approach, h) Reconstructed color image from image (g), i) Grey-level image with Campisi et al. approach, j) Reconstructed color image from image (i). 


\section{Conclusion}

In this paper, we have presented an efficient method to protect the colors of images by giving a free access to the corresponding gray level images. From a gray level image with a hidden color palette, if you have a secret key then it is possible to reconstruct a color image. A user does not need to download the color image. Once the key is bought the user can view the color image but just with the help of the gray level image and the key. The proposed method consists of three main steps, involving the quantization of the color image, color reordering by finding an index image and the data-hiding in order to hide the color information in the index image. The second step is to find an index image which is very close to the gray level image. In the step to embed the color palette within the index image, the main objective is to create the minimum possible distortion. This work has been done in the framework of a project to provide limited access to the private digital painting database of the Louvre Museum of Paris, France. We applied our approach to several digital paintings and we have illustrated and analyzed the obtained results as a function of the number of colors for the quantization and as a function of the size of the layer to build the path. The obtained results are convincing and encourage us to continue in this way.

In particular, we are thinking of increasing the visual and statistical invisibility using adaptive steganography (Pevny et al., 2010), and analyzing possible attacks, without a key, in order to reconstruct the color image (Chaumont and Puech, 2008).

\section{Acknowledgment}

This investigation was in part supported by the TSAR projects which is a French national project ANR-05-SSIA0017-05 of the ANR ARA SSIA (Agence Nationale de la Recherche, Action de Recherche Amont, Sécurité Systèmes Embarqués et Intelligence Ambiante). We would like also to thank officials of the Louvre Museum for access to the digital paintings and for valuable discussions.

\section{References}

Ball, G. H., Hall, D. J., Jun. 1966. ISODATA, a Novel Method of Data Analysis and Pattern Classification. In: Proc. of the International Communication Conference.

Battiato, S., Rundo, F., Stanco, F., Dec. 2007. Self Organizing Motor Maps for Color-Mapped Image Re-Indexing. IEEE Trans. on Image Processing 16 (12), 2905-2915.

Bender, W., Gruhl, D., Morimoto, N., Lu, A., 1996. Techniques for Data Hiding. I.B.M. Systems Journal 35 (3-4), 313-336.

Bernarding, J., Thiel, A., Grzesik, A., 2001. A JAVA-Based DICOM Server with Integration of Clinical Findings and DICOM-Conform Data Encrytion. International Journal of Medical Informatics 64, 429-438.

Campisi, P., Kundur, D., Hatzinakos, D., Neri, A., 2002. Compressive Data Hiding: An Unconventional Approach for Improved Color Image Coding. EURASIP Journal on Applied Signal Processing 2002 (2), 152-163.

Cappellini, V., Piva, A., September 2006. Opportunities and Issues of Image Processing for Cultural Heritage Applications. In: Proc. 14th European Signal Processing Conference (EUSIPCO 06). Firenze, Italy.

Castagno, R., Sodomaco, A., Oct. 1998. Estimation of Image Feature Reliability for an Interactive Video Segmentation Scheme. In: Proc. IEEE Int. Conf. on Image Processing. Vol. 1. Chicago, USA, pp. 938-942.
Cayre, F., Fontaine, C., Furon, T., 2005. Watermarking Security: Theory and Practice. IEEE Transactions on Signal Processing 53 (10), 3976-3987, special issue "Supplement on Secure Media III".

Chang, C., Hwang, M., Chen, T.-S., 2001. A New Encryption Algorithm for Image Cryptosystems. The Journal of Systems and Software 58, 83-91.

Chaumont, M., Puech, W., June 2006. A Color Image Hidden in a Grey-Level Image. In: Proc. 3rd European Conference on Colour in Graphics, Imaging and Vision (CGIV06). Leeds, UK, pp. 226-231.

Chaumont, M., Puech, W., Jan. 2007. A Fast and Efficient Method to Protect Color Images. In: IS\&T/SPIE 19th Annual Symposium on Electronic Imaging, Visual Communications and Image Processing, VCIP'2007, SPIE'2007. Vol. 6508. San Jose, California, USA.

Chaumont, M., Puech, W., Jun. 2008. Attack By Colorization of a Grey-Level Image Hiding its Color Palette. In: IEEE International Conference on Multimedia \& Expo, ICME'2008. Hannover, Germany.

Chung, K., Chang, L., 1998. Large Encrypting Binary Images with Higher Security. Pattern Recognition Letters 19, 461-468.

Dunn, J. C., 1974. A Fuzzy Relative of the ISODATA Process and its Use in Detecting Compact Well-Separated Clusters. Journal of Cybernetics 3, 3257.

Eskicioglu, A., Delp, E., 2001. An Overview of Multimedia Content Protection in Consumer Electronics Devices. Signal Processing: Image Communication 16 (7), 681-699.

Fridrich, J., April 1999. A New Steganographic Method for Palette Images. In: IS\&T PICS. Savannah, Georgia, USA, pp. 285-289.

Fridrich, J., 2009. Steganography in Digital Media: Principles, Algorithms, and Applications, 1st Edition. Cambridge University Press, New York, NY, USA.

Fridrich, J., Goljan, M., 2002. Practical Steganalysis: State-of-the-Art. In: Proc. of SPIE Photonics West, Electronic Imaging 2002. Vol. 4675. pp. 1-13.

Gervautz, M., Purgathofer, W., 1990. A Simple Method for Color Quantization: Octree Quantization. Graphics Gems, A.S. Glassner, 287-293.

Horiuchi, T., Noharaa, F., Tominaga, S., Nov. 2010. Accurate Reversible Colorto-Gray Mapping Algorithm Without Distortion Conditions. Pattern Recognition Letters 31 (15).

Kerckhoffs, A., 1883. La cryptographie militaire. Journal des sciences militaires 9, 5-38.

Ko, K.-W., Kwon, O.-S., Son, C.-H., Ha, Y.-H., Feb. 2008. Color Embedding and Recovery Based on Wavelet Packet Transform. Journal of Imaging Science and Technology 52 (1).

Kodovský, J., Fridrich, J., Feb. 2012. Steganalysis of JPEG images using rich models. In: Media Watermarking, Security, and Forensics III, Part of IS\&T/SPIE 22th Annual Symposium on Electronic Imaging, SPIE'2012. Vol. 8303. San Francisco, California, USA.

Lemma, A., Katzenbeisser, S., Celik, M., van der Veen, M., 2006. Secure Watermark Embedding through Partial Encryption. In: International Workshop on Digital Watermarking (IWDW 2006). Vol. 4283. Springer Lecture Notes in Computer Science, pp. 433-445.

Lian, S., Liu, Z., Zhen, R., Weng, H., 2006. Commutative Watermarking and Encryption for Media Data. Optical Engineering 45 (8), 080510-1-080510 3.

Memon, N., Venkateswaran, A., Nov. 1996. On Ordering Color Maps for Lossless Predictive Coding. IEEE Trans. on Image Processing 5 (11), 15221527.

Nikolaidis, N., Pitas, I., 1998. Robust Image Watermarking in the Spatial Domain. Signal Processing 66 (3), 385-403.

Norcen, R., Podesser, M., Pommer, A., Schmidt, H., Uhl, A., 2003. Confidential Storage and Transmission of Medical Image Data. Computers in Biology and Medicine 33, 277-292.

Pevny, T., Filler, T., Bas, P., 2010. Using High-Dimensional Image Models to Perform Highly Undetectable Steganography. In: Bohme, R., Fong, P. W. L., Safavi-Naini, R. (Eds.), Information Hiding. Vol. 6387 of Lecture Notes in Computer Science. Springer, pp. 161-177.

Pinho, A., Neves, A., Nov. 2004. A Survey on Palette Reordering Methods for Improving the Compression of Color-Indexed Images. IEEE Trans. on Image Processing 13 (11), 1411-1418.

Puech, W., Chaumont, M., Strauss, O., January 2008. A Reversible Data Hiding Method for Encrypted Images. In: Proc. SPIE, Electronic Imaging, Security, Forensics, Steganography, and Watermarking of Multimedia Contents X. Vol. 6819. San Jose, CA, USA, pp. 68191E-1-68191E-9.

Puech, W., Coatrieux, G., may 2008. Chapter 10: Coding: Encryption- 
Watermarking-Compression for Medical Information Security. Compression of Biomedical Images and Signals, A. Naït-Ali and Christine CavaroMenard, Digital Signal Processing, ISTE-Wiley.

Quach, T.-T., Feb. 2012. Locatability of modified pixels in steganographic images . In: Media Watermarking, Security, and Forensics III, Part of IS\&T/SPIE 22th Annual Symposium on Electronic Imaging, SPIE'2012. Vol. 8303. San Francisco, California, USA.

R. De Queiroz, Mar. 2010. Reversible Color-to-Gray Mapping Using Subband Domain Texturization. Pattern Recognition Letters, 20th SIBGRAPI: Advances in Image Processing and Computer Vision 31 (4), 269-276.

R. De Queiroz and K. Braun, 2006. Color to Gray and Back: Color Embedding Into Textured Gray Images. IEEE Trans. on Image Processing 15 (6), 14641470.

Schneier, B., 1995. Applied cryptography. Wiley, New-York, USA

Shih, F. Y., Wu, S. Y., 2003. Combinational Image Watermarking in the Spatial and Frequency Domains. Pattern Recognition 36, 969-975.

Sinha, A., Singh, K., April 2003. A Technique for Image Encryption Using Digital Signature. Optics Communications 218 (4-6), 229-234.

Tzeng, C., Yang, Z., Tsai, W., 2004. Adaptative Data Hiding in Palette Images by Color Ordering and Mapping With Security Protection. IEEE Trans. on Communications 52 (5), 791-800.

Uhl, A., Pommer, A., 2005. Image and Video Encryption: From Digital Rights Management to Secured Personal Communication. Springer.

Wu, M.-Y., Ho, Y.-K., Lee, J.-H., 2003. An Iterative Method of Palette-Based Image Steganography. Pattern Recognition Letters 25, 301-309.

Xu, X., Dexter, S., Eskicioglu, A., January 2004. A Hybrid Scheme for Encryption and Watermarking. In: Wong, P., Delp, E. (Eds.), Proc. of SPIE, Electronic Imaging, Security and Watermarking of Multimedia Contents VI. Vol. 5306. SPIE, IS\&T, San Jose, CA, USA, pp. 725-736.

Zhao, Y., Campisi, P., Kundur, D., 2004. Dual Domain for Authentication and Compression of Cultural Heritage Images. IEEE Trans. on Image Processing 13 (3), 430-448 